\title{
Drivers and barriers to fruit and vegetable consumption in adults: a qualitative research synthesis
}

\author{
S. Howard \\ KBS University of Kent, Canterbury, Kent CT2 7TZ, UK
}

\begin{abstract}
Many factors affect fruit and vegetable consumption and these have been explored extensively by quantitative methods. Systematic reviews of quantitative research on fruit and vegetables consumption have collated psychological factors in adults, concluding that self efficacy, social support and knowledge are important behaviour predictors ${ }^{(1)}$. While, another found behaviour was predicted by habit, motivation and goals, self-efficacy, knowledge and taste ${ }^{(2)}$. A review of environmental factors showed the impact of income on consumption $^{(3)}$. To date only one review has included qualitative research of studies on children and adults finding availability of fruit and vegetables was important ${ }^{(4)}$.

A qualitative research synthesis was conducted on studies researching fruit and vegetable consumption in adults to evaluate the drivers and barriers that impact on fruit and vegetable consumption in adults. The synthesis was conducted on fifty-six studies. Data extraction was conducted with thematic analysis due to its flexibility for various methods and philosophical approaches ${ }^{(5)}$. Categories were developed from the data and sectioned into the biopsychosocial model ${ }^{(6)}$. More barriers were present in the biological section for taste, texture and satiety. For psychological factors drivers were health (specific, general, health of others), habit, meaningful events to the self and nutrition information, while barriers were time, effort, knowledge of fruit and vegetables, cooking skills, preference, mood, attitude and self-efficacy. Social drivers included having a garden, childhood experiences, women as gatekeepers, tradition, support of peers and information, while barriers were cost, availability, family preferences, access, waste and quality.

These data provide an overview of qualitative research showing complex constructs operate within fruit and vegetable consumption. Findings differ from the results of quantitative systematic reviews, offering greater and richer understanding of drivers and barriers that could be used to improve the development of interventions to increase fruit and vegetable consumption and subsequent health of the population.
\end{abstract}

1. Shaikh A, Yaroch A, Nebeling L et al. (2008) Psychosocial predictors of fruit and vegetable consumption in adults a review of the literature. Am J Prev Med 34, 535-543.

2. Guillaumie L, Godin G \& Vezina-Im LA (2010) Psychosocial determinants of fruit and vegetable intake in adult population: a systematic review. Int J Behav Nutr Phys Act (Review).

3. Kamphuis CB, Giskes K de Bruijn GL et al. (2006) Environmental determinants of fruit and vegetable consumption among adults: a systematic review. Br J Nutr 96, 620-635.

4. Jago R, Baronowski T \& Baronowski J (2007) Fruit and vegetable availability: a micro environmental mediating variable? Public Health Nutr 10, 681-689.

5. Braun V \& Clarke V (2006) Using thematic analysis in psychology. Qual Res Psychol 3, 77-101.

6. Engel GL The need for a new medical model: a challenge for biomedicine. Science 196, 29-36. 